\title{
Estimation of the gray level variations in soft and hard peri-implant tissue from $X$-ray images
}

\author{
"C.C.Leung ${ }^{1}$, P.C.K.Kwok ${ }^{1}$, K.Y.Zee ${ }^{2}$, F.H.Y.Chan ${ }^{1}$ \\ ${ }^{1}$ Department of Electrical \& Electronic Engineering \\ ${ }^{2}$ Department of Periodontology \\ The University of Hong Kong, Pokfulam Road, Hong Kong \\ "E-mail: ccleung@eee.hku.hk
}

\begin{abstract}
In the treatment and assessment of Periodontal disease. The Periapical films are taken at regular intervals. The films are compared around the region of the implant to monitor the changes that have taken place. A computer-assisted method to automate this process is presented in this paper. To compare two images, a pair of $\mathrm{X}$ ray images are superimposed on top of each other. The images are normalized and transformed to obtain the best fit. These images are subtracted and the difference in pixel values are used as a basis for analysis. A method for improving the alignment accuracy and contrast compensation is highlighted in this paper.
\end{abstract}

Keywords: Periodontal disease, Periapical film, X-ray image, Image alignment.

\section{Introduction}

Estimation of gray level variations in peri-implant tissue has been used widely in Periodontology in diagnosing the condition of the bones and tissues around the dental implants. The traditional three-step method [1-3] starts by taking a series of X-ray films at a few months' intervals. By superimposing the reference film against a grid, reference image is partitioned into small rectangular blocks. The resulting image is captured using a CCD camera. A followup X-ray film is then positioned manually on top of the reference film and the grid to obtain the best fit for a given rectangular block. The reference film is then removed and the follow-up film is digitized against the grid. Gray level subtraction can then be performed by software for the two images. The differences are grouped and counted manually. There are three limitations of this conventional method. (a) Piece-wise alignment cannot ensure a good fit for all pixel positions within the block. (b) The alignment procedure is subjective and the result cannot be easily replicated. (c) Manual piece-wise alignment is time consuming and prone to errors.

In order minimize the errors and inconsistencies of the above method, the estimation process should be performed by computer. A computer assisted alignment method based on image analysis technique is presented here. The method consists of (1) Image normalization : to correct the difference in contrast and brightness of the two images captured from the Periapical films; (2) Boundary detection of features that are known to be time invariant. (3) Image alignment : using the boundaries of the time invariant features as a reference. The method is described in detail in the next section.

\section{The Method}

The Hardware Set-up. The X-ray films are captured using a high precision 3-CCD color video camera (JVC KY-F55). The camera is interfaced to a Data Translation Fidelity 200 frame grabber board installed in a PC. The image is digitized into 8-bit gray scale format. Image analysis is performed by using MATLAB (The Math Works Inc.).

\section{Image Analysis.}

(1) Image normalization. As a result of slight differences in exposure time, lighting and the conditions during film development, there will be a mismatch in the overall contrast and brightness between the two X-ray films taken at different times. Brightness and contrast corrections have to be performed.

Consider two X-ray films taken 6 months apart. The original (older) image $P$ is used as the reference. The newer image $Q$, taken at a later time, will have its gray levels transformed to match to the original image. For reliable results, it is necessary to identify two regions corresponding to features that are known to be time invariant. For example, as in figure 2 , the artificial tooth represents a bright region and the oral cavity represents a dark region. Choosing a bright region and a dark region will enable a linear function to be constructed for the mapping of gray levels from the newer image to the original image.

Furthermore the regions should be chosen carefully so that the bright region is not clipped to peak white while the dark region is not be clipped to the black level on both the newer and the original images. Otherwise, the mapping will not be meaningful. 
The central part of the artificial tooth is chosen as the bright region and the central part of the oral cavity is chosen as the dark region. In both cases, there is a substantial area with an almost constant luminance level. This enables reliable averages to be computed for the regions.

Let $p_{2}, p_{1}$ be the average gray levels of the bright and dark regions respectively in the original image; and $q_{2}, q_{1}$ be the average gray levels of the bright and dark regions respectively in the newer image. Using a linear model (Figure 1), the gray level $q$ of the newer image $Q$ will be normalized to a gray level $p$ according to the following equation:

$p-p_{1} / p_{2}-p_{1}=q-q_{1} / q_{2}-q_{1}$

(2) Boundary detection. The purpose of boundary detection is to identify reliable reference points so that alignment can be performed. As shown in Figure 2, the boundary between the implant and the surrounding tissue is very distinct and does not vary with time. Thus the boundary can be used as a reliable reference.

To establish the boundary, simple thresholding is first applied to both the original and the newer images. The following description applies to the original image $P$. Let $f_{p}(x, y)$ be the gray level of a pixel at coordinates $(x, y)$ in the original image. The thresholded image is binary and a pixel at coordinates $(x, y)$ can assume a value $h_{p}(x, y)$ equal to 0 or 1 according to:

$h_{p}(x, y)=\left\{\begin{array}{l}1, f(x, y) \geq T \\ 0, f(x, y)<T\end{array}\right.$

where $T$ is the threshold value, chosen as the mid point between the average gray levels of the implant and the tissue.

Thresholding is followed by edge detection. Many techniques have been investigated and compared. The Laplacian method was chosen and it is the best for images with low as well as high contrast.

The Laplacian operator expressed in vector form is

$$
L[h(x, y)]=w^{\prime} \eta
$$

where $w$ is a vector of coefficients of the mask, and $\eta$ is a vector of pixel values under the mask. The gradient $\mathrm{G}[f(\mathrm{x}, \mathrm{y})]$ at $(\mathrm{x}, \mathrm{y})$ can assume one of three level,

$$
s(x, y)=\left\{\begin{array}{c}
0, i f G[f(x, y)]<T \\
+, i f G[f(x, y)] \geq T, L[f(x, y)] \geq 0 \\
-, i f G[f(x, y)] \geq T, L[f(x, y)]<0
\end{array}\right.
$$

Non edge pixels will have a gradient of 0 . Pixels on the dark and bright sides of the edge take on values of " + "and "-" respectively. Figure $3 a$ shows an example of edge detection applied to the boundary between the implant and the tissue.

Similarly the above boundary detection procedure is applied to the newer image $Q$.

(3). Image alignment. Two reference points are selected from the edge between the implant and the tissue. As shown in Figure $3 \mathrm{a}$, the screw threads are very distinct, time invariant and therefore reliable features most suited for the process of alignment. The first and the fifth threads are chosen as reference points. A straight line is drawn between the two points as illustrated in Figure $3 b$. The line $L_{Q}$ obtained from the newer image and the line $L_{P}$ from the original images can then be used to estimate the rotation and translation that need to be applied to the newer image $Q$ to obtain the best fit.

After alignment, gray level subtraction between the aligned newer image $Q^{\prime}$ and the original image $P$ can be obtained on a pixel by pixel basis, i.e.,

$\operatorname{Difference}(x, y)=Q^{\prime}(x, y)-P(x, y)$

for the pixels in the tissue surrounding the implant. Statistics can then be gathered for automatic analysis in the same way as in the conventional method [5].

\section{Results and Discussion}

Figure 4 shows the difference image, obtained from the same example in Figures 2 and 3 . The software correctly identify the positions of the edges. After image alignment, the fitting is the best it can achieve given the limited resolution of the images. The set-up has been evaluated and used by a dental researchers who have been using the traditional manual method for quite some time. The researchers found the new set-up very simple and convenient to use. The gray level distribution of three normal subjects in the control group are shown in figure 5. This result is obtained from the Prince Philip Dental Hospital, the typical distribution for the normal subject demonstrating the validity of this new method. According to user opinion, this new method can achieve better and more consistent results than with the 
manual method, taking much less time to complete the procedure than the traditional approach.

\section{Conclusion}

The semi-automatic method presented in this paper accomplishes all the objectives that were set out: accuracy, consistency and speed. It saves the human operator (researcher) a lot of time and effort in an attempt to align two X-ray films. It is superior than the manual method in its repeatability and alignment accuracy.

\section{References}

[1] I.Fourmousis, U.Bragger, W.Burgin, M.Tonetti, N.P.Lang. Digital image processing I Evaluation of gray level correction methods in vitro. Clin. Oral Impl. Res., p.1-11, September 1993.

[2] H.G.Grondahl, K.Grondahl, R.L.Webber. A digital subtraction technique for dental radiography. Oral Surg. Vol. 55, Number 1, p.96-102, January 1983.

[3] E.H.Verdonschot, A.J.Sanders, A.J.Plasschaert. A computer-aided image analysis system for area measurement of tooth root surfaces. J. Periodontol., Vol. 61, Number 5, p.275-280, May 1990.

[4] R.C.Gonzalez, P.Wintz. Digital Image Processing. Addison Wesley Publishing Co., 1987.

[5] K.A.Kornman. Nature of periodontal diseases: assessment and diagnosis. J. Periodontal Research, Vol.22, p.192-204.

\section{The normalization chart}

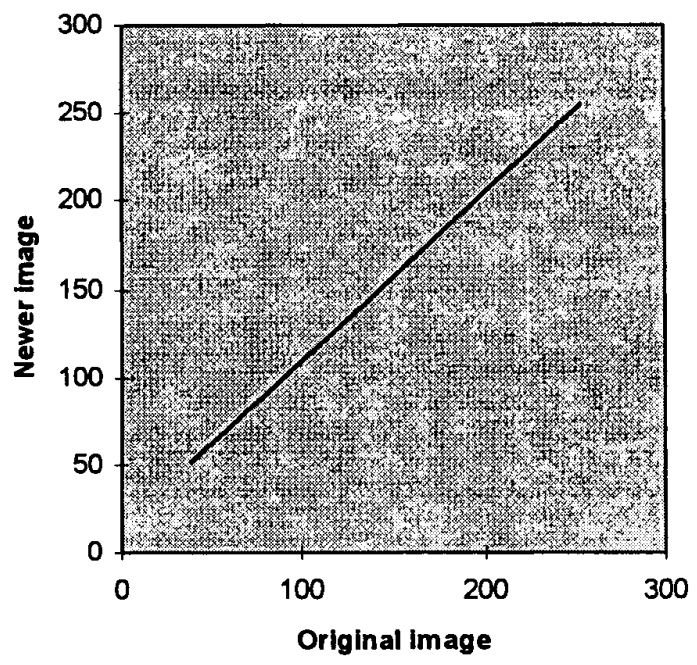

Figure 1 The linear model calculated by original and followup images.

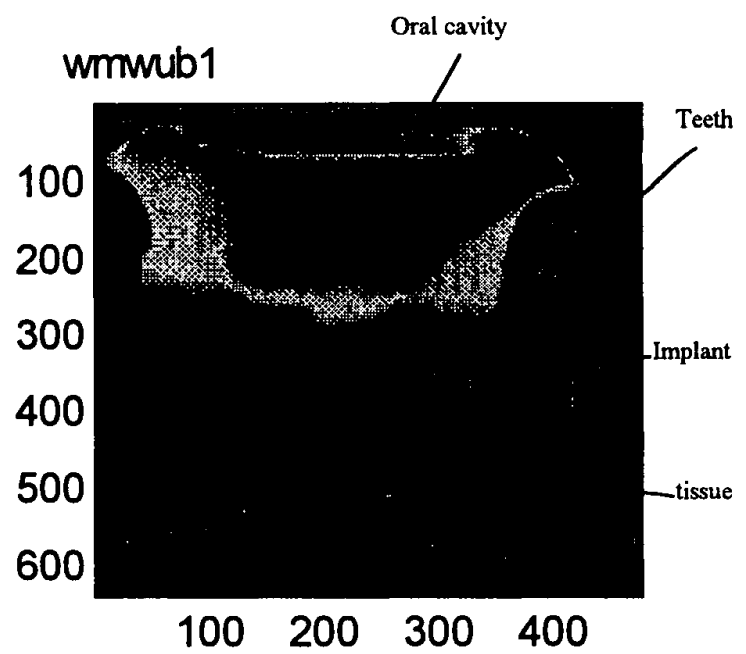

Figure 2. The Periapical film, the image contains the tooth, tissue and implant. In boundary detection, implant is the best region selected as the reference to do the thresholding because of its significant brightness. 


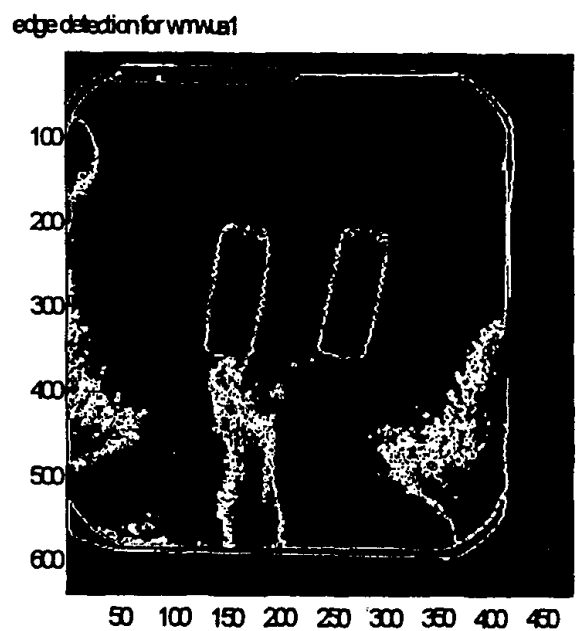

(a)

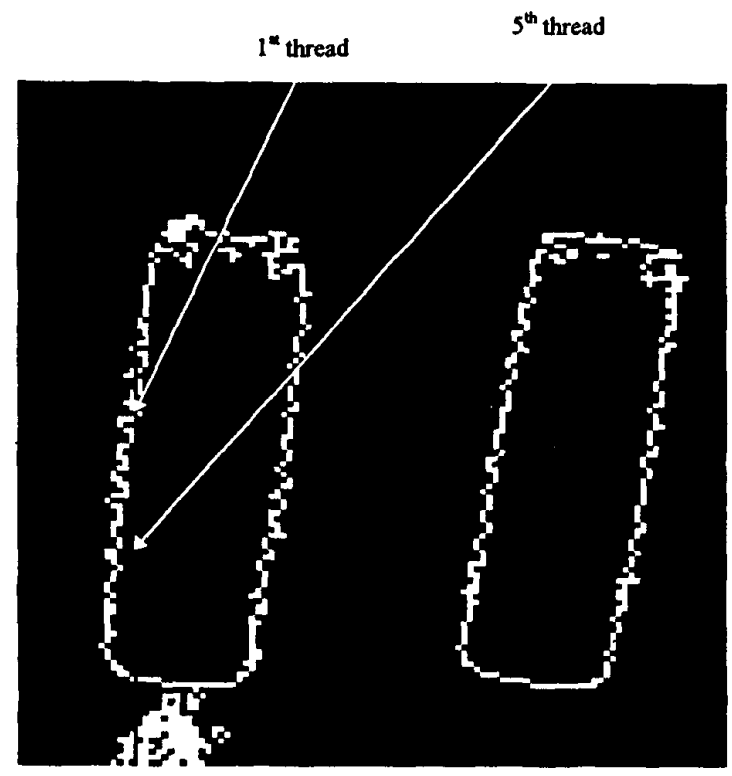

(b)

Figure 3. (a) After the edge detection using thresholding and Laplacian method, the boundary of the reference (Artificial tooth) can be seen in the image, and (b) the two reference points plot in the $1^{\text {st }}$ thread and $5^{\text {th }}$ thread in the Artificial tooth.

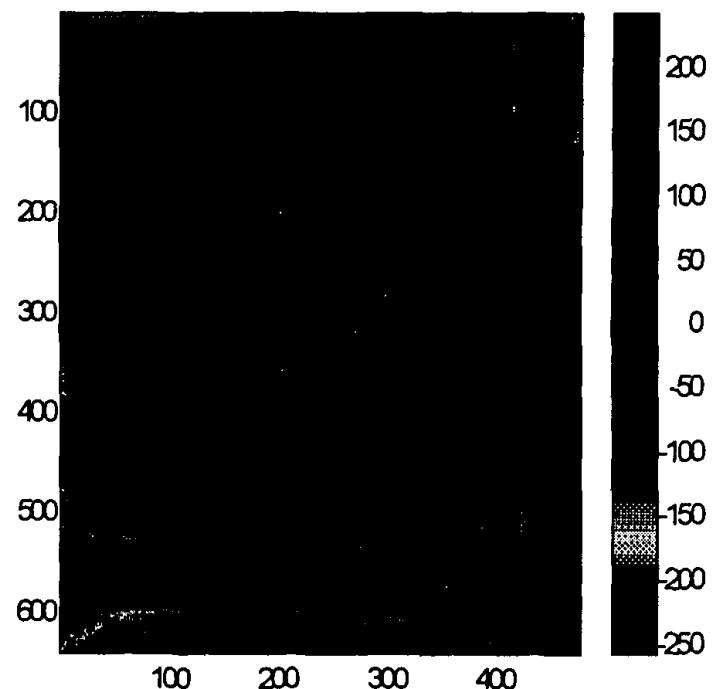

Figure 4. The difference image. The difference in reference (Artificial tooth) is approximately to zero and the average pixel value of interest region is around \pm 7 .

\section{Distribution of the group for the gray level number}

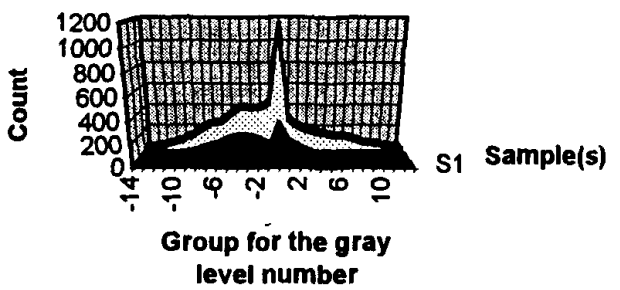

Figure 5. The distribution of gray levels in three normal subjects, giving typical distribution for normal subjects counted in the same interest region. The distribution range is around \pm 7 gray levels (measured at Prince Philip Dental Hospital in Hong Kong). 